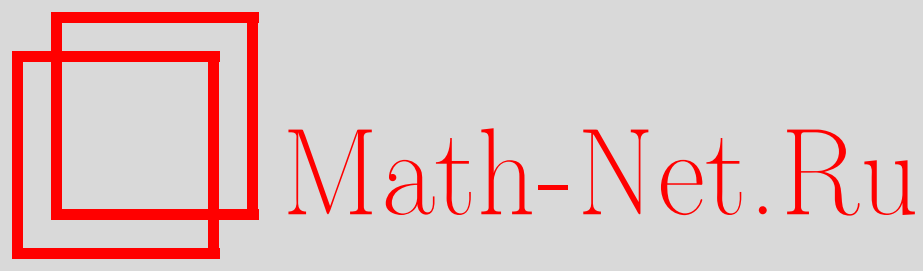

A. В. Латышев, А. А. Юшканов, Температурный скачок в вырожденных квантовых газах с энергией возбуждения Боголюбова и при наличии конденсата БозеЭйнштейна, ТМФ, 2010, том 165, номер 1, 145-159

DOI: https://doi.org/10.4213/tmf6568

Использование Общероссийского математического портала Math-Net.Ru подразумевает, что вы прочитали и согласны с пользовательским соглашением http://www . mathnet.ru/rus/agreement

Параметры загрузки:

IP: 34.229 .45 .116

26 апреля 2023 г., 14:41:18

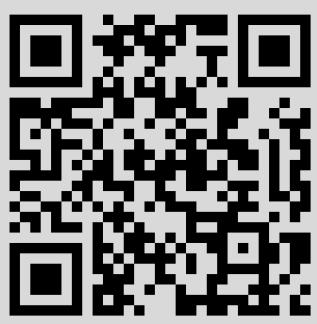




\title{
ТЕМПЕРАТУРНЫЙ СКАЧОК В ВЫРОЖДЕННЫХ КВАНТОВЫХ ГАЗАХ С ЭНЕРГИЕЙ ВОЗБУЖДЕНИЯ БОГОЛЮБОВА И ПРИ НАЛИЧИИ КОНДЕНСАТА БОЗЕ-ЭЙНШТЕЙНА
}

\begin{abstract}
Построено линеаризованное кинетическое уравнение, моделирующее поведение вырожденного квантового бозе-газа с частотой столкновений, зависящей от импульса элементарных возбуждений. Рассмотрен общий случай зависимости энергии элементарных возбуждений от импульса согласно формуле Боголюбова. Получено аналитическое решение полупространственной граничной задачи о скачке температуры на границе вырожденного бозе-газа при наличии конденсата Бозе-Эйнштейна. Получено выражение для сопротивления Капицы.
\end{abstract}

Ключевые слова: вырожденный квантовый бозе-газ, конденсат Бозе-Эйнштейна, скачок температуры, интеграл столкновений.

\section{1. ВВЕДЕНИЕ}

Неравновесные свойства ограниченных в объеме квантовых газов вызывают в последнее время значительный интерес. В частности, важное значение имеет такое явление, как скачок температуры [1] на границе газа и конденсированного (в частности, твердого) тела при наличии нормального к поверхности теплового потока. Такой скачок температуры в квантовых жидкостях часто называют скачком температуры Капицы [2].

Задача о скачке температуры является одной из важнейших в кинетической теории [3]. Аналитическое решение этой задачи для случая разреженного одноатомного газа получено в работе [4]. Задача о скачке температуры для электронного газа в металле рассматривалась в наших работах [5], [6]. В этих работах получено аналитическое решение задачи о скачке температуры, обусловленном потоком тепла к поверхности.

Поведение квантовых газов в последнее время вызывает повышенный интерес. Это связано, в частности, с развитием экспериментальных методик получения и исследования квантовых газов при экстремально низких температурах [7]. В большинстве работ исследуются объемные свойства квантовых газов [8], [9]. В то же время

* Московский государственный областной университет, Москва, Россия. E-mail: avlatyshev@mail.ru,yushkanov@inbox.ru 
очевидна важность учета граничных эффектов при изучении свойств таких систем. Отметим работу [10], в которой рассматриваются термодинамически равновесные свойства квантовых газов в полупространстве.

До сих пор скачок температуры Капицы вычислялся при учете только рассеяния фононов на границе между двумя средами, а рассеянием фононов в объеме пренебрегалось [11]. В работе [12] рассматривалась задача о скачке температуры в квантовом ферми-газе. Было получено аналитическое решение при произвольной степени вырождения газа. В работе [13] рассматривалась аналогичная задача для бозе-газа. Однако газ предполагался невырожденным, т.е. отсутствовал конденсат Бозе-Эйнштейна [14].

Настоящая работа посвящена анализу задачи о скачке температуры в вырожденном бозе-газе. Наличие конденсата Бозе-Эйнштейна приводит к существенной модификации как постановки задачи, так и метода ее решения. При этом для описания кинетических процессов вблизи поверхности используется кинетическое уравнение с модельным интегралом столкновений. Предполагается, что граничные условия на поверхности носят зеркально-диффузный характер. Рассматривается кинетическое уравнение, в котором согласно теории Боголюбова для бозе-газа взята общая зависимость энергии элементарных возбуждений от импульса. Характер рассеяния элементарных возбуждений на поверхности учитывается при этом путем введения феноменологического коэффициента зеркальности рассеяния на поверхности.

В работе [15] пренебрегалось наличием фононной компоненты в формуле Боголюбова, а в работе [16], наоборот, считалось, что фононная компонента преобладает в элементарных возбуждениях бозе-газа. В данной работе рассматривается общий случай зависимости энергии элементарных возбуждений от импульса.

\section{2. ВЫВОД КИНЕТИЧЕСКОГО УРАВНЕНИЯ}

Для описания поведения газа мы будем использовать кинетическое уравнение с модельным интегралом столкновений, аналогичное тому, которое используется для описания классического газа. При этом будет проведен необходимый учет квантового характера бозе-газа и наличия конденсата Бозе-Эйнштейна.

Для разреженного бозе-газа эволюция функции распределения частиц газа $f$ описывается кинетическим уравнением [14]

$$
\frac{\partial f}{\partial t}+\frac{\partial \mathcal{E}}{\partial \mathbf{p}} \nabla f=I(f),
$$

где $\mathcal{E}$ - кинетическая энергия частиц газа, $\mathbf{p}-$ их импульс, $I(f)$ - интеграл столкновений.

В случае вырожденного бозе-газа при кинетическом описании необходимо учитывать, что свойства конденсата Бозе-Эйнштейна могут меняться в зависимости от пространственных и временно́й координат, т. е., по сути дела, необходимо рассматривать двухжидкостную модель (точнее, “двухфлюидную”, так как рассматривается не жидкость, а газ). Обозначим плотность конденсата Бозе-Эйнштейна через $\rho_{\text {cond }}=\rho_{\text {cond }}(\mathbf{r}, t)$, а его скорость через $\mathbf{u}_{\text {cond }}=\mathbf{u}_{\text {cond }}(\mathbf{r}, t)$. Тогда для плотностей 
потока массы $\mathbf{j}$, энергии $\mathbf{Q}$ и импульса $\Pi_{i k}$ конденсата Бозе-Эйнштейна (в предположении нулевого химического потенциала) будем иметь следующие выражения [11]:

$$
\mathbf{j}=\rho_{\text {cond }} \mathbf{u}_{\text {cond }}, \quad \mathbf{Q}=\frac{\rho_{\text {cond }} u_{\text {cond }}^{2}}{2} \mathbf{u}_{\text {cond }}, \quad \Pi_{i k}=\rho_{\text {cond }} u_{\text {cond }, i} u_{\text {cond }, k} .
$$

Законы сохранения числа частиц, энергии и импульса требуют в стационарном случае выполнения соотношений

$$
\nabla \mathbf{j}=-\int I(f) d \Omega_{\mathrm{B}}, \quad \nabla \mathbf{Q}=-\int \mathcal{E}(\mathbf{p}) I(f) d \Omega_{\mathrm{B}}, \quad \nabla \Pi=-\int \mathbf{p} I(f) d \Omega_{\mathrm{B}},
$$

где

$$
d \Omega_{\mathrm{B}}=\frac{(2 s+1) d^{3} p}{(2 \pi \hbar)^{3}},
$$

$s$ - спин частицы, $\mathcal{E}(\mathbf{p})$ - энергия, $\hbar$ - постоянная Планка, $I(f)$ - интеграл столкновений из уравнения (1).

В дальнейшем нас будет интересовать случай движения с малыми (по сравнению с тепловыми) скоростями. Отметим, что для конденсата Бозе-Эйнштейна величины $\mathbf{Q}$ и $\Pi_{i k}$ зависят от скорости нелинейно (пропорциональны третьей и второй степеням скорости). Поэтому в линейном приближении по скорости $\mathbf{u}_{\text {cond }}$ законы сохранения энергии и импульса можно записать в виде

$$
\int \mathcal{E}(\mathbf{p}) I(f) d \Omega_{\mathrm{B}}=0, \quad \int \mathbf{p} I(f) d \Omega_{\mathrm{B}}=0 .
$$

Для слабовзаимодействующего бозе-газа согласно теории Боголюбова справедливо следующее соотношение для энергии возбуждений $\mathcal{E}(p)[14]$ :

$$
\mathcal{E}(p)=\left[u_{0}^{2} p^{2}+\left(\frac{p^{2}}{2 m}\right)^{2}\right]^{1 / 2}, \quad u_{0}=\left(\frac{4 \pi \hbar^{2} a n}{m^{2}}\right)^{1 / 2},
$$

где $a$ - длина рассеяния молекул газа, $n$ - концентрация, $m$ - масса частиц газа, $\mathbf{p}$ - импульс элементарных возбуждений, $u_{0}$ - скорость звука. Параметр $a$ характеризует силу взаимодействия молекул газа. Для слабовзаимодействующего газа этот параметр можно считать малым.

В нашей предыдущей работе [15] рассмотрен случай, когда для достаточно малых значений величины $a$ выполняется соотношение $u_{0}^{2} \ll k T / m, k$ - постоянная Больцмана, $T$ - температура. В этом случае первым слагаемым в квадратных скобках в формуле (2) можно пренебречь. Выражение для энергии $\mathcal{E}(p)$ приобретет тот же вид, что и в случае невзаимодействующих молекул: $\mathcal{E}(p)=p^{2} / 2 m$. В этом случае $\partial \mathcal{E}(p) / \partial \mathbf{p}=\mathbf{v}$.

В работе [16] рассмотрен случай, когда в выражении (2) превалирует фононная компонента, т.е. когда $T \ll m u_{0}^{2} / k$. В этом случае согласно $(2)$ получаем, что $\mathcal{E}(p)=$ $u_{0}|\mathbf{p}|$. Следовательно, $\partial \mathcal{E}(p) / \partial \mathbf{p}=u_{0} \mathbf{p} / p$.

Теперь рассмотрим общий случай, когда ни первым, ни вторым слагаемым в элементарных возбуждениях нельзя пренебречь. В этом случае

$$
\frac{\partial \mathcal{E}(p)}{\partial \mathbf{p}}=\alpha(p) \mathbf{p}
$$


где

$$
\alpha(p)=\frac{u_{0}^{2}+p^{2} /\left(2 m^{2}\right)}{\mathcal{E}(p)} .
$$

При рассмотрении кинетического уравнения (1) под частицами газа необходимо понимать элементарные возбуждения бозе-газа со спектром энергий (2). Характер элементарных возбуждений проявляется в свойствах интеграла столкновений. Возьмем в уравнении (1) в качестве интеграла столкновений его $\tau$-приближение. Тогда характер элементарных возбуждений будет отражаться в зависимости частоты столкновений от импульса возбуждений [14], [1]:

$$
\frac{\partial f}{\partial t}+\alpha(p) \mathbf{p} \frac{\partial f}{\partial \mathbf{r}}=\nu\left(\mathbf{p}-\mathbf{p}_{0}\right)\left(f_{\mathrm{B}}^{*}-f\right) .
$$

Здесь $f$ - функция распределения, $\nu\left(\mathbf{p}-\mathbf{p}_{0}\right)=\nu_{0}\left|\mathbf{p}-\mathbf{p}_{0}\right|^{\gamma}-$ зависимость частоты столкновений от импульса возбуждений, $\gamma$ - постоянная (в случае, например, когда в элементарных возбуждениях превалирует фононная компонента, величина $\gamma \geqslant 3[14]), \mathbf{p}_{0}=m \mathbf{v}_{0}$, где $\mathbf{v}_{0}-$ скорость нормальной компоненты бозе-газа, $f_{\mathrm{B}}^{*}-$ равновесная функция распределения Бозе-Эйнштейна,

$$
f_{\mathrm{B}}^{*}=\left[e^{\mathcal{E}\left(\mathbf{p}-\mathbf{p}_{*}\right) /\left(k T_{*}\right)}-1\right]^{-1},
$$

$\nu_{0}$ - параметр модели, $\nu_{0}=\nu_{1} /\left(m k T_{\mathrm{S}}\right)^{1 / 2}$, величина $\nu_{1}$ имеет смысл обратной средней длины свободного пробега $l, \nu_{1} \sim 1 / l, T_{\mathrm{s}}$ - температура газа в некоторой точке поверхности,

$$
\mathcal{E}\left(\mathbf{p}-\mathbf{p}_{*}\right)=\left[u_{0}^{2}\left(\mathbf{p}-\mathbf{p}_{*}\right)^{2}+\left(\frac{\left(\mathbf{p}-\mathbf{p}_{*}\right)^{2}}{2 m}\right)^{2}\right]^{1 / 2} .
$$

Параметры, входящие в $f_{\mathrm{B}}^{*}$, а именно $T_{*}=T_{*}(\mathbf{r}, t)$ и $\mathbf{p}_{*}=\mathbf{p}_{*}(\mathbf{r}, t)$, определяются из требования выполнения законов сохранения импульса и энергии:

$$
\int \nu\left(\mathbf{p}-\mathbf{p}_{0}\right) \mathbf{p}\left[f-f_{\mathrm{B}}^{*}\right] d^{3} p=0, \quad \int \nu\left(\mathbf{p}-\mathbf{p}_{0}\right) \mathcal{E}(\mathbf{p})\left[f-f_{\mathrm{B}}^{*}\right] d^{3} p=0 .
$$

Эти параметры будем называть соответственно эффективной температурой и импульсом.

Закон сохранения числа частиц здесь не выполняется, так как часть частиц переходит в конденсат Бозе-Эйнштейна.

Будем считать, что скорость газа много меньше средней тепловой скорости, а возникающие характерные перепады температуры на длине свободного пробега $l$ малы по сравнению с температурой газа. В этих предположениях возможна линеаризация задачи. Перейдем к линеаризации уравнения (3).

Начнем с линеаризации эффективной температуры:

$$
T_{*}=T_{\mathrm{s}}+\delta T_{*}=T_{\mathrm{s}}\left(1+\frac{\delta T_{*}}{T_{\mathrm{s}}}\right) .
$$

Функция распределения Бозе-Эйнштейна является функцией импульса $\mathbf{p}$ и параметров $\mathbf{p}_{*}$ и $\delta T_{*} / T_{\mathrm{s}}$. Ее линеаризацию проведем по двум последним параметрам:

$$
f_{\mathrm{B}}^{*}\left(\mathbf{p}, \mathbf{p}_{*}, \frac{\delta T_{*}}{T_{\mathrm{s}}}\right)=f_{\mathrm{B}}^{*}(\mathbf{p}, 0,0)+\frac{\partial f_{\mathrm{B}}^{*}}{\partial \mathbf{p}_{*}}(\mathbf{p}, 0,0) \mathbf{p}_{*}+\frac{\partial f_{\mathrm{B}}^{*}}{\partial\left(\delta T_{*} / T_{\mathrm{s}}\right)}(\mathbf{p}, 0,0) \frac{\delta T_{*}}{T_{\mathrm{s}}} .
$$


В результате такой линеаризации получаем

$$
f_{\mathrm{B}}^{*}\left(\mathbf{p}, \mathbf{p}_{*}, \frac{\delta T_{*}}{T_{\mathrm{S}}}\right)=f_{\mathrm{B}}(p)+g(p) \alpha(p) \frac{\mathbf{p} \mathbf{p}_{*}}{k T_{\mathrm{S}}}+g(p) \frac{\mathcal{E}(p)}{k T_{\mathrm{S}}} \frac{\delta T_{*}}{T_{\mathrm{S}}},
$$

где

$$
f_{\mathrm{B}}(p) \equiv f_{\mathrm{B}}^{*}(\mathbf{p}, 0,0)=\frac{1}{e^{\mathcal{E}(p) /\left(k T_{\mathrm{s}}\right)}-1}, \quad g(p)=\frac{e^{\mathcal{E}(p) /\left(k T_{\mathrm{s}}\right)}}{\left[e^{\mathcal{E}(p) /\left(k T_{\mathrm{s}}\right)}-1\right]^{2}} .
$$

Линеаризацию функции распределения согласно (5) проведем следующим образом:

$$
f(\mathbf{r}, \mathbf{p}, t)=f_{\mathrm{B}}(p)+g(p) h(\mathbf{r}, \mathbf{p}, t)
$$

Из (5) и (6) находим

$$
f_{\mathrm{B}}-f=g(p)\left[\alpha(p) \frac{\mathbf{p p}_{*}}{k T_{\mathrm{S}}}+\frac{\mathcal{E}(p)}{k T_{\mathrm{s}}} \frac{\delta T_{*}}{T_{\mathrm{s}}}\right] .
$$

Вернемся к уравнению (3). Выполним линеаризацию этого уравнения согласно (5). Заметим, что в линейном приближении величину $\nu\left(\mathbf{p}-\mathbf{p}_{0}\right)$ в уравнении (3) можно заменить на $\nu(p)=\nu_{0} p^{\gamma}$. Тогда уравнение (3) в результате линеаризации имеет вид

$$
\frac{\partial h}{\partial t}+\alpha(p) \mathbf{p} \frac{\partial h}{\partial \mathbf{r}}=\nu_{0} p^{\gamma}\left[\alpha(p) \frac{\mathbf{p p}_{*}}{k T_{\mathrm{s}}}+\frac{\mathcal{E}(p)}{k T_{\mathrm{s}}} \frac{\delta T_{*}}{T_{\mathrm{s}}}\right] .
$$

В уравнении (7) введем безразмерный импульс (скорость) $\mathbf{C}=\mathbf{v} / v_{T}=\mathbf{p}_{T} / p_{T}$, где $v_{T}=\sqrt{k T_{\mathrm{s}} / m}-$ тепловая скорость частиц газа, $\mathbf{p}_{T}-$ их импульс. Тогда $\mathcal{E}(p)=$ $k T_{\mathrm{s}} \mathcal{E}(C)$, где $\mathcal{E}(C)=\sqrt{w_{0}^{2} C^{2}+C^{4} / 4}$, а $w_{0}=u_{0} / v_{T}$ - безразмерная скорость звука. Кроме того, заметим, что $\alpha(p)=\alpha(C) / m$, где

$$
\alpha(C)=\frac{w_{0}^{2}+C^{2} / 2}{\sqrt{w_{0}^{2} C^{2}+\left(C^{2} / 2\right)^{2}}} .
$$

Введем безразмерные время $\tau=\nu_{0} t$ и координату $\mathbf{r}_{1}=\nu_{0} \sqrt{m /\left(k T_{\mathrm{s}}\right)} \mathbf{r}$. Уравнение $(3)$ в безразмерных переменных принимает вид

$$
\frac{\partial h}{\partial \tau}+\alpha(C) \mathbf{C} \frac{\partial h}{\partial \mathbf{r}_{1}}=C^{\gamma}\left[\alpha(C) \mathbf{C C}_{*}\left(\mathbf{r}_{1}, \tau\right)+\mathcal{E}(C) \frac{\delta T_{*}}{T_{\mathrm{s}}}\left(\mathbf{r}_{1}, \tau\right)-h\left(\mathbf{r}_{1}, \mathbf{C}, \tau\right)\right] .
$$

В законах сохранения (4) также проведем линеаризацию. В результате получаем законы сохранения импульса и энергии в следующей форме:

$$
\begin{gathered}
\int p^{\gamma} \mathbf{p}\left[\alpha(p) \frac{\mathbf{p} \mathbf{p}_{*}}{k T_{\mathrm{s}}}+\frac{\mathcal{E}(p)}{k T_{\mathrm{s}}} \frac{\delta T_{*}}{T_{\mathrm{s}}}-h\left(\mathbf{r}_{1}, \mathbf{p}, \tau\right)\right] g(p) d^{3} p=0, \\
\int p^{\gamma} \mathcal{E}(p)\left[\alpha(p) \frac{\mathbf{p} \mathbf{p}_{*}}{k T_{\mathrm{s}}}+\frac{\mathcal{E}(p)}{k T_{\mathrm{S}}} \frac{\delta T_{*}}{T_{\mathrm{s}}}-h\left(\mathbf{r}_{1}, \mathbf{p}, \tau\right)\right] g(p) d^{3} p=0 .
\end{gathered}
$$

В этих равенствах выполним интегрирование по безразмерному импульсу:

$$
\begin{array}{r}
\int C^{\gamma} \mathbf{C}\left[\alpha(C) \mathbf{C} \mathbf{C}_{*}+\mathcal{E}(C) \frac{\delta T_{*}}{T_{\mathrm{s}}}-h\left(\mathbf{r}_{1}, \mathbf{C}, \tau\right)\right] g(C) d^{3} C=0, \\
\int C^{\gamma} \mathcal{E}(C)\left[\alpha(C) \mathbf{C C}_{*}+\mathcal{E}(C) \frac{\delta T_{*}}{T_{\mathrm{s}}}-h\left(\mathbf{r}_{1}, \mathbf{C}, \tau\right)\right] g(C) d^{3} C=0,
\end{array}
$$


где

$$
g(C)=\frac{e^{\mathcal{E}(C)}}{\left(e^{\mathcal{E}(C)}-1\right)^{2}} .
$$

Из этих законов сохранения энергии и импульса находим

$$
\begin{aligned}
\mathbf{C}_{*}\left(\mathbf{r}_{1}, \tau\right) & =\frac{\int C^{\gamma} \mathbf{C} h\left(\mathbf{r}_{1}, \mathbf{C}, \tau\right) g(C) d^{3} C}{\int C^{\gamma} C_{x}^{2} \alpha(C) g(C) d^{3} C}, \\
\frac{\delta T_{*}}{T_{\mathrm{s}}}\left(\mathbf{r}_{1}, \tau\right) & =\frac{\int C^{\gamma} \mathcal{E}(C) h\left(\mathbf{r}_{1}, \mathbf{C}, \tau\right) g(C) d^{3} C}{\int C^{\gamma} \mathcal{E}^{2}(C) g(C) d^{3} C} .
\end{aligned}
$$

Вычислим интегралы, стоящие в знаменателях двух последних равенств. Имеем

$$
\int C^{\gamma} C_{x}^{2} \alpha(C) g(C) d^{3} C=\frac{4 \pi}{3} g_{1}, \quad \int C^{\gamma} \mathcal{E}^{2}(C) g(C) d^{3} C=4 \pi g_{2},
$$

где

$$
g_{1}=\int_{0}^{\infty} C^{\gamma+4} \alpha(C) g(C) d C, \quad g_{2}=\int_{0}^{\infty} C^{\gamma+2} \mathcal{E}^{2}(C) g(C) d C .
$$

Таким образом, параметры уравнения окончательно имеют вид

$$
\begin{aligned}
\mathbf{C}_{*}\left(\mathbf{r}_{1}, \tau\right) & =\frac{3}{4 \pi g_{1}} \int C^{\gamma} \mathbf{C h}\left(\mathbf{r}_{1}, \mathbf{C}, \tau\right) g(C) d^{3} C, \\
\frac{\delta T_{*}}{T_{\mathrm{s}}}\left(\mathbf{r}_{1}, \tau\right) & =\frac{1}{4 \pi g_{2}} \int C^{\gamma} \mathcal{E}(C) h\left(\mathbf{r}_{1}, \mathbf{C}, \tau\right) g(C) d^{3} C .
\end{aligned}
$$

С помощью равенств (9) представим уравнение (8) в стандартной для теории переноса форме:

$$
\frac{\partial h}{\partial \tau}+\alpha(C) \mathbf{C} \frac{\partial h}{\partial \mathbf{r}_{1}}+C^{\gamma} h\left(\mathbf{r}_{1}, \mathbf{C}, \tau\right)=\frac{C^{\gamma}}{4 \pi} \int K\left(\mathbf{C}, \mathbf{C}^{\prime}\right) h\left(\mathbf{r}_{1}, \mathbf{C}^{\prime}, \tau\right) C^{\prime \gamma} g\left(C^{\prime}\right) d^{3} C^{\prime},
$$

где

$$
K\left(\mathbf{C}, \mathbf{C}^{\prime}\right)=\frac{3 \alpha(C) \mathbf{C C}^{\prime}}{g_{1}}+\frac{\mathcal{E}(C) \mathcal{E}\left(C^{\prime}\right)}{g_{2}} .
$$

\section{3. ПОСТАНОВКА ЗАДАЧИ}

В рассматриваемой задаче вырожденный бозе-газ занимает полупространство $x>0$ над плоской поверхностью, с которой происходит теплообмен между конденсированной фазой и газом. Поэтому далее функцию $h$ можно рассматривать в виде $h\left(\tau, \mathbf{r}_{1}, \mathbf{C}\right)=h(x, \mu, C)$. Здесь $\mu$ есть косинус угла между направлением вектора скорости $\mathbf{C}$ и осью $x$ в сферической системе скоростей бозе-частиц, $C_{x}=\mu C$. Уравнение (10) для функции $h$ запишется в виде

$$
\mu \frac{\alpha(C)}{C^{\gamma-1}} \frac{\partial h}{\partial x}+h(x, \mu, C)=\frac{3 \alpha(C) C \mu}{2 g_{1}} W_{1}(x)+\frac{\mathcal{E}(C)}{2 g_{2}} W_{2}(x),
$$

где

$$
W_{1}(x)=\int_{-1}^{1} \int_{0}^{\infty} C^{\prime \gamma+3} \mu^{\prime} h\left(x, \mu^{\prime}, C^{\prime}\right) g\left(C^{\prime}\right) d \mu^{\prime} d C^{\prime}
$$




$$
W_{2}(x)=\int_{-1}^{1} \int_{0}^{\infty} \mathcal{E}\left(C^{\prime}\right) h\left(x, \mu^{\prime}, C^{\prime}\right) C^{\prime \gamma+2} g\left(C^{\prime}\right) d \mu^{\prime} d C^{\prime} .
$$

Задача состоит в нахождении величины относительного скачка температуры $\varepsilon_{T}=\Delta T / T_{\mathrm{s}}, \Delta T=T_{\mathrm{s}}-T$, как функции $Q_{x}$ - величины проекции на ось $x$ потока тепла. Учитывая линейный характер задачи, можно записать $\varepsilon_{T}=R Q_{x}$. Безразмерный коэффициент $R$ в выражении для скачка температуры называется сопротивлением Капицы.

Очевидно, что уравнение (11) имеет следующие частные решения: $h_{1}(x, \mu, C)=$ $\alpha(C) C \mu$ и $h_{2}(x, \mu, C)=\mathcal{E}(C)$. Следовательно, функцией распределения Чепмена-Энскога является функция

$$
h_{\mathrm{as}}(x, \mu, C)=B^{+} \alpha(C) C \mu-\varepsilon_{T} \mathcal{E}(C),
$$

где величина $B^{+}$пропорциональна величине потока тепла $Q_{x}$.

Теперь, предполагая отражение элементарных возбуждений от стенки зеркально-диффузным, сформулируем граничные условия:

$$
\begin{gathered}
h(0, \mu, C)=q h(0,-\mu, C), \quad 0<\mu<1, \\
h(x, \mu, C)=B^{+} \alpha(C) C \mu-\varepsilon_{T} \mathcal{E}(C)+o(1), \quad x \rightarrow+\infty, \quad-1<\mu<0 .
\end{gathered}
$$

Итак, задача состоит в решении уравнения (11) с граничными условиями (12). Особый интерес представляет нахождение величины температурного скачка $\varepsilon_{T}$.

\section{4. СВЕДЕНИЕ К ИНТЕГРАЛЬНОМУ УРАВНЕНИЮ}

Продолжим функцию $h$ в полупространство $x<0$ симметричным образом:

$$
h(x, \mu, C)=h(-x,-\mu, C) .
$$

Тогда при $x<0$ распределение Чепмена-Энскога имеет вид

$$
h_{\mathrm{as}}(x, \mu, C)=B^{-} \alpha(C) C \mu-\varepsilon_{T} \mathcal{E}(C),
$$

причем $B^{+}=-B^{-}$. Теперь выделим у функции $h$ составляющую, представляющую собой распределение Чепмена-Энскога, полагая, что

$$
h(x, \mu, C)=B^{ \pm} \alpha(C) C \mu-\varepsilon_{T} \mathcal{E}(C)+h_{\mathrm{c}}(x, \mu, C), \quad \text { если } \quad \pm x>0 .
$$

Сформулируем граничные условия для функции $h_{\mathrm{c}}$ для нижнего и верхнего полупространств:

$$
\begin{array}{rlrl}
h_{\mathrm{c}}(+0, \mu, C)= & -(1+q) B^{+} \alpha(C) C \mu+ & \\
& +(1-q) \varepsilon_{T} \mathcal{E}(C)+q h_{\mathrm{c}}(+0,-\mu, C), \quad 0<\mu<1, \\
h_{\mathrm{c}}(-0, \mu, C)= & -(1+q) B^{-} \alpha(C) C \mu+ & \\
& +(1-q) \varepsilon_{T} \mathcal{E}(C)+q h_{\mathrm{c}}(-0,-\mu, C), \quad-1<\mu<0, \\
h_{\mathrm{c}}(+\infty, \mu, C)= & 0, \quad h_{\mathrm{c}}(-\infty, \mu, C)=0 .
\end{array}
$$


Вставим эти граничные условия в кинетическое уравнение. Получаем следующее уравнение:

$$
\begin{aligned}
\mu \frac{\partial h_{\mathrm{c}}}{\partial x} & +\frac{C^{\gamma-1}}{\alpha(C)} h_{\mathrm{c}}(x, \mu, C)=\frac{C^{\gamma-1}}{\alpha(C)}\left\{\frac{3 \alpha(C) C \mu}{2 g_{1}} W_{1}(x)+\frac{\mathcal{E}(C)}{2 g_{2}} W_{2}(x)+\right. \\
& \left.+|\mu|\left[-(1+q) B^{+} \alpha(C) C|\mu|+(1-q) \varepsilon_{T} \mathcal{E}(C)-(1-q) h_{\mathrm{c}}(\mp 0, \mu, C)\right] \delta(x)\right\}
\end{aligned}
$$

где $\delta(x)$ - дельта-функция Дирака.

Уравнение (13) фактически объединяет два уравнения. Дело в том, что слагаемое $h_{\mathrm{c}}(-0, \mu, C)$ отвечает положительным значениям переменной $\mu$ : $0<\mu<1$, a слагаемое $h_{\mathrm{c}}(+0, \mu, C)$ - отрицательным значениям $\mu$ : $-1<\mu<0$.

Решение уравнений (13) будем искать в виде интегралов Фурье:

$$
\begin{aligned}
h_{\mathrm{c}}(x, \mu, C) & =\frac{1}{2 \pi} \int_{-\infty}^{\infty} e^{i k x} \Phi(k, \mu, C) d k, & \delta(x) & =\frac{1}{2 \pi} \int_{-\infty}^{\infty} e^{i k x} d k, \\
W_{1}(x) & =\frac{1}{2 \pi} \int_{-\infty}^{\infty} e^{i k x} E_{1}(k) d k, & W_{2}(x) & =\frac{1}{2 \pi} \int_{-\infty}^{\infty} e^{i k x} E_{2}(k) d k .
\end{aligned}
$$

Начнем с поиска неизвестных граничных значений $h_{\mathrm{c}}(\mp 0, \mu, C)$. Выразим эти значения через спектральные плотности $E_{1}(k)$ и $E_{2}(k)$. Для этого будем рассматривать уравнение (13) при $x<0$ и $x>0$. В обоих случаях слагаемое с дельта-функцией выпадает из уравнения (13). Решая сначала уравнение (13) при $x>0, \mu<0$, считая известной правую часть этого уравнения и удовлетворяя граничным условиям вдали от стенки, получаем

$$
h_{\mathrm{c}}^{+}(x, \mu, C)=-\frac{1}{\mu} \exp \left(-\frac{x}{\mu} \frac{C^{\gamma-1}}{\alpha(C)}\right) \int_{x}^{+\infty} \exp \left(\frac{t}{\mu} \frac{C^{\gamma-1}}{\alpha(C)}\right) W(t, \mu, C) d t,
$$

где

$$
W(t, \mu, C)=\frac{C^{\gamma-1}}{\alpha(C)}\left[\frac{3 \alpha(C) C \mu}{2 g_{1}} W_{1}(t)+\frac{\mathcal{E}(C)}{2 g_{2}} W_{2}(t)\right] .
$$

При $x<0, \mu>0$ аналогично получаем

$$
h_{\mathrm{c}}^{-}(x, \mu, C)=\frac{1}{\mu} \exp \left(-\frac{x}{\mu} \frac{C^{\gamma-1}}{\alpha(C)}\right) \int_{-\infty}^{x} \exp \left(\frac{t}{\mu} \frac{C^{\gamma-1}}{\alpha(C)}\right) W(t, \mu, C) d t .
$$

Подчеркнем, что в уравнении (13) граничные значения функции $h_{\mathrm{c}}( \pm 0, \mu, C)$ являются граничными значениями приведенных выше функций $h_{\mathrm{c}}^{ \pm}(x, \mu, C)$ при $x \rightarrow \pm 0$ из соответствующих полуплоскостей. Из двух последних равенств для интегралов Фурье вытекает, что

$$
W(t, \mu, C)=\frac{C^{\gamma-1}}{\alpha(C)}\left[\frac{3 \alpha(C) C \mu}{2 g_{1}} \cdot \frac{1}{2 \pi} \int_{-\infty}^{\infty} e^{i k t} E_{1}(k) d k+\frac{\mathcal{E}(C)}{2 g_{2}} \cdot \frac{1}{2 \pi} \int_{-\infty}^{\infty} e^{i k t} E_{2}(k) d k\right] .
$$

После несложных вычислений в (14) находим

$$
\begin{aligned}
h_{\mathrm{c}}^{+}(x, \mu, C)= & \frac{C^{2(\gamma-1)}}{2 \pi} \int_{-\infty}^{+\infty} e^{i k x}\left[\frac{3 \alpha(C) C \mu}{2 g_{1}} E_{1}(k)+\right. \\
& \left.+\frac{\mathcal{E}(C)}{2 g_{2}} E_{2}(k)\right] \frac{d k}{C^{2(\gamma-1)}+k^{2} \mu^{2} \alpha^{2}(C)} .
\end{aligned}
$$


Аналогично можно показать, что для функции $h_{\mathrm{c}}^{-}(x, \mu, C)$ получается точно такое же выражение. Следовательно, учитывая четность по $k$ функций $E_{1}(k)$ и $E_{2}(k)$, получаем

$$
h_{\mathrm{c}}^{ \pm}(0, \mu, C)=\frac{C^{\gamma}}{\pi} \int_{0}^{+\infty}\left[\frac{3 \alpha(C) C \mu}{2 g_{1}} E_{1}(k)+\frac{\mathcal{E}(C)}{2 g_{2}} E_{2}(k)\right] \frac{d k}{C^{2(\gamma-1)}+k^{2} \mu^{2} \alpha^{2}(C)} .
$$

Итак, граничные значения искомой функции $h_{\mathrm{c}}( \pm 0, \mu, C)$ из уравнения (13) являются функциями $h_{\mathrm{c}}^{ \pm}(0, \mu, C)$, определенными равенством $(15)$.

Из соотношения (15) видно, что два уравнения (13) можно объединить в одно:

$$
\begin{aligned}
& \frac{\mu \alpha(C)}{C^{\gamma-1}} \frac{\partial h_{\mathrm{c}}}{\partial x}+h_{\mathrm{c}}(x, \mu, C)=\frac{3 \alpha(C) C \mu}{2 g_{1}} W_{1}(x)+\frac{\mathcal{E}(C)}{2 g_{2}} W_{2}(x)+ \\
& \quad+|\mu|\left\{-(1+q) B^{+}|\mu| \alpha(C) C+(1-q) \varepsilon_{T} \mathcal{E}(C)-(1-q) h_{\mathrm{c}}^{ \pm}(0, \mu, C)\right\} \delta(x) .
\end{aligned}
$$

\section{5. ХАРАКТЕРИСТИЧЕСКАЯ СИСТЕМА УРАВНЕНИЙ}

Перейдем в уравнении (16) к интегралам Фурье. Получаем уравнение

$$
\begin{gathered}
{\left[C^{\gamma}+i k \mu \alpha(C) C\right] \Phi(k, \mu, C)=\frac{3 \alpha(C) C^{\gamma+1} \mu}{2 g_{1}} E_{1}(k)+\frac{\mathcal{E}(C) C^{\gamma}}{2 g_{2}} E_{2}(k)-} \\
-(1+q) B^{+} \mu^{2} C^{\gamma}+(1-q) \varepsilon_{T} C^{\gamma+1}|\mu|-(1-q)|\mu| \frac{C^{3 \gamma}}{\pi} \times \\
\quad \times \int_{0}^{\infty}\left[\frac{3 \alpha(C) C \mu}{2 g_{1}} E_{1}(k)+\frac{\mathcal{E}(C)}{2 g_{2}} E_{2}(k)\right] \frac{d k}{C^{2 \gamma}+k^{2} \mu^{2} \alpha^{2}(C) C^{2}}
\end{gathered}
$$

Несложно найти выражения для $E_{1}(k)$ и $E_{2}(k)$ :

$$
\begin{aligned}
& E_{1}(k)=\int_{-1}^{1} \int_{0}^{\infty} C^{\gamma+3} \mu \Phi(k, \mu, C) g(C) d \mu d C, \\
& E_{2}(k)=\int_{-1}^{1} \int_{0}^{\infty} \mathcal{E}(C) C^{\gamma+2} \Phi(k, \mu, C) g(C) d \mu d C .
\end{aligned}
$$

Выразим функцию $\Phi(k, \mu, C)$ из уравнения (17) и подставим его в последние два выражения. Получим характеристическую систему уравнений, которую запишем в векторной форме:

$$
\Lambda(k) E(k)=2(1+q) B^{+} T_{1}(k)-2(1-q) \varepsilon_{T} T_{2}(k)+\frac{1-q}{\pi} \int_{0}^{\infty} J\left(k, k_{1}\right) E\left(k_{1}\right) d k_{1},
$$

где введены следующие обозначения: $\Lambda(k)$ - дисперсионная матрица-функция, $E(k)$ - неизвестный вектор-столбец,

$$
\Lambda(k)=\left(\begin{array}{ll}
\frac{3 k^{2}}{g_{1}} T_{\gamma+6,4}^{3,0}(k) & \frac{i k}{g_{2}} T_{2 \gamma+4,2}^{1,1}(k) \\
\frac{3 i k}{g_{1}} T_{2 \gamma+4,2}^{2,1}(k) & \frac{k^{2}}{g_{2}} T_{\gamma+4,2}^{2,2}(k)
\end{array}\right), \quad E(k)=\left(\begin{array}{c}
E_{1}(k) \\
E_{2}(k)
\end{array}\right)
$$


$T_{1}(k)$ и $T_{2}(k)$ - вектор-столбцы свободных членов,

$$
\begin{gathered}
T_{1}(k)=\left(\begin{array}{c}
i k T_{2 \gamma+4,4}^{1,0}(k) \\
-T_{3 \gamma+2,2}^{0,1}(k)
\end{array}\right), \quad T_{2}(k)=\left(\begin{array}{c}
i k T_{2 \gamma+5,3}^{1,0}(k) \\
-T_{3 \gamma+3,1}^{0,1}(k)
\end{array}\right), \\
T_{m, n}^{r, s}(k)=\int_{0}^{1} \int_{0}^{\infty} \frac{\alpha^{r}(C) \mathcal{E}^{s}(C) C^{m} \mu^{n}}{C^{2 \gamma}+k^{2} \mu^{2} \alpha^{2}(C) C^{2}} d \mu d C, \quad r, s=0,1,2, \ldots,
\end{gathered}
$$

$J\left(k, k_{1}\right)$ - матричное ядро интегрального уравнения (17),

$$
J\left(k, k_{1}\right)=\left(\begin{array}{cc}
-\frac{3}{g_{1}} J_{5 \gamma+4,3}^{1,0}\left(k, k_{1}\right) & \frac{i k}{g_{2}} J_{4 \gamma+4,3}^{1,1}\left(k, k_{1}\right) \\
\frac{3 i k}{g_{1}} J_{4 \gamma+4,3}^{2,1}\left(k, k_{1}\right) & -\frac{1}{g_{2}} J_{5 \gamma+2,1}^{0,2}\left(k, k_{1}\right)
\end{array}\right) .
$$

В матрице $J\left(k, k_{1}\right)$ введены интегралы

$$
J_{m, l}^{r, s}\left(k, k_{1}\right)=\int_{0}^{1} \int_{0}^{\infty} \frac{\alpha^{r}(C) \mathcal{E}^{s}(C) C^{m} \mu^{l} g(C) d \mu d C}{\left(C^{2 \gamma}+k^{2} \mu^{2} \alpha^{2}(C) C^{2}\right)\left(C^{2 \gamma}+k_{1}^{2} \mu^{2} \alpha^{2}(C) C^{2}\right)}
$$

\section{6. МЕТОД ПОСЛЕДОВАТЕЛЬНЫХ ПРИБЛИЖЕНИЙ}

Решение уравнения (18) будем искать в виде

$$
\begin{aligned}
\varepsilon_{T} & =\frac{1+q}{1-q}\left[\varepsilon_{0}+\varepsilon_{1}(1-q)+\varepsilon_{2}(1-q)^{2}+\cdots\right], \\
E(k) & =2(1+q)\left[E^{(0)}(k)+E^{(1)}(k)(1-q)+E^{(2)}(k)(1-q)^{2}+\cdots\right] .
\end{aligned}
$$

Подставим эти равенства в характеристическое уравнение. Получим счетную систему уравнений:

$$
\begin{aligned}
& \Lambda(k) E^{(0)}(k)=-B^{+} T_{1}(k)+\varepsilon_{0} T_{2}(k), \\
& \Lambda(k) E^{(1)}(k)=\varepsilon_{1} T_{2}(k)-\frac{1}{\pi} \int_{0}^{\infty} J\left(k, k_{1}\right) E^{(0)}\left(k_{1}\right) d k_{1}, \\
& \Lambda(k) E^{(2)}(k)=\varepsilon_{2} T_{2}(k)-\frac{1}{\pi} \int_{0}^{\infty} J\left(k, k_{1}\right) E^{(1)}\left(k_{1}\right) d k_{1}, \quad \ldots
\end{aligned}
$$

Определитель дисперсионной матрицы назовем дисперсионной функцией: $\lambda(z) \equiv$ $\operatorname{det} \Lambda(z)=k^{2} \omega(k)$, где

$$
\omega(k)=\frac{3}{g_{1} g_{2}}\left[k^{2} T_{\gamma+4,6}^{3,0}(k) T_{\gamma+4,2}^{2,2}(k)+T_{2 \gamma+4,2}^{1,1}(k) T_{2 \gamma+4,2}^{2,1}(k)\right] .
$$

Обратная к дисперсионной матрица такова: $\Lambda^{-1}(k)=D(k) / \lambda(k)$, где

$$
D(k)=\left(\begin{array}{cc}
\frac{k^{2}}{g_{2}} T_{\gamma+4,2}^{2,2}(k) & -\frac{i k}{g_{2}} T_{2 \gamma+4,2}^{1,1}(k) \\
-\frac{3 i k}{g_{1}} T_{2 \gamma+4,2}^{2,1}(k) & \frac{3 k^{2}}{g_{1}} T_{\gamma+4,6}^{3,0}(k)
\end{array}\right) .
$$


Рассмотрим построение рядов (19). Введем обозначение:

$$
E^{(m)}(k)=\left(\begin{array}{l}
E_{1}^{(m)}(k) \\
E_{2}^{(m)}(k)
\end{array}\right), \quad m=0,1,2, \ldots
$$

Из уравнения (20) находим

$$
\begin{aligned}
E_{1}^{(0)}(k)= & \frac{i k}{\omega(k) g_{2}} T_{\gamma+4,2}^{2,2}(k)\left[B^{+} T_{2 \gamma+4,4}^{1,0}(k)-\varepsilon_{0} T_{2 \gamma+5,3}^{1,0}(k)\right]+ \\
& +\frac{i}{k \omega(k) g_{2}} T_{2 \gamma+4,2}^{1,1}(k)\left[B^{+} T_{3 \gamma+2,2}^{0,1}(k)-\varepsilon_{0} T_{3 \gamma+3,1}^{0,1}(k)\right], \\
E_{2}^{(0)}(k)= & \frac{3}{\omega(k) g_{1}} T_{2 \gamma+4,2}^{2,1}(k)\left[B^{+} T_{2 \gamma+4,4}^{1,0}(k)-\varepsilon_{0} T_{2 \gamma+5,3}^{1,0}(k)\right]+ \\
& +\frac{3}{\omega(k) g_{1}} T_{\gamma+4,6}^{3,0}(k)\left[-B^{+} T_{3 \gamma+2,2}^{0,1}(k)+\varepsilon_{0} T_{3 \gamma+3,1}^{0,1}(k)\right] .
\end{aligned}
$$

Величина $E_{2}^{(0)}(k)$ существует при всех значениях $k$, но величина $E_{1}^{(0)}(k)$ имеет в нуле простой полюс. Для его устранения возьмем $\varepsilon_{0}$ в виде

$$
\varepsilon_{0}=B^{+} \frac{T_{3 \gamma+2,2}^{0,1}(0)}{T_{3 \gamma+3,1}^{0,1}(0)}=B^{+} \frac{g_{\varepsilon, 2}(\gamma)}{3 g_{\varepsilon, 3}(\gamma)},
$$

где

$$
\begin{aligned}
g_{\varepsilon, 2}(\gamma) & =\int_{0}^{\infty} \mathcal{E}(C) C^{\gamma+2} g(C) d C, & g_{\varepsilon, 3}(\gamma) & =\int_{0}^{\infty} \mathcal{E}(C) C^{\gamma+3} g(C) d C, \\
\mathcal{E}(C) & =\sqrt{w_{0}^{2} c^{2}+\left(\frac{C^{2}}{2}\right)^{2}}, & g(C) & =\frac{e^{\mathcal{E}(C)}}{\left(e^{\mathcal{E}(C)}-1\right)^{2}} .
\end{aligned}
$$

Выражение для $\varepsilon_{0}$ подставим в $(23)$ и (24), а затем подставим выражения (23) и (24) в $(21)$ и находим $E^{(1)}(k)$. Далее подставим $E^{(1)}(k)$ в уравнение $(22)$ и находим $E^{(2)}(k)$. Продолжая этот процесс неограниченно, построим все члены рядов (19).

\section{7. СКАЧОК ТЕМПЕРАТУРЫ И СОПРОТИВЛЕНИЕ КАПИЦЫ}

Найдем величину $\varepsilon_{0}$ в явном виде. Величина $B^{+}$пропорциональна величине потока тепла:

$$
\mathbf{Q}=\int f(x, \mathbf{p}) \frac{\partial \mathcal{E}(p)}{\partial \mathbf{p}} \mathcal{E}(p) d \Omega_{\mathrm{B}} .
$$

Преобразуем это выражение:

$$
\mathbf{Q}=\frac{(2 s+1) u_{0}^{2}}{(2 \pi \hbar)^{3}} \int h(x, \mathbf{p}) g(p) \alpha(p) \mathbf{p} \mathcal{E}(p) d^{3} p,
$$

а затем проинтегрируем его по безразмерному импульсу. Для этого заметим, что

$$
\alpha(p) \mathbf{p} \mathcal{E}(p) d^{3} p=\frac{k T_{\mathrm{s}}}{m}\left(k T_{\mathrm{s}} m\right)^{2} \alpha(C) \mathbf{C} \mathcal{E}(C) .
$$


В результате получим

$$
\mathbf{Q}=\frac{(2 s+1)\left(k T_{\mathrm{s}}\right)^{3} m}{(2 \pi \hbar)^{3}} \int h(x, \mathbf{C}) \alpha(C) \mathbf{C E}(C) g(C) d^{3} C .
$$

Заменим здесь функцию $h$ на ее разложение Чепмена-Энскога $h_{\text {as }}$. В результате для $x$-компоненты потока тепла имеем

$$
\begin{aligned}
Q_{x}= & \frac{(2 s+1)\left(k T_{\mathrm{s}}\right)^{3} m}{(2 \pi \hbar)^{3}} \int_{-1}^{1} \int_{0}^{\infty} \int_{0}^{2 \pi}\left[B^{+} \alpha(C) C \mu-\varepsilon_{T} \mathcal{E}(C)\right] \times \\
& \times \alpha(C) C \mu \mathcal{E}(C) C^{2} g(C) d \mu d C d \chi=\frac{(2 s+1)\left(k T_{\mathrm{s}}\right)^{3} m}{(2 \pi \hbar)^{3}} \cdot \frac{4 \pi}{3} g_{\alpha \varepsilon}(\gamma) B^{+},
\end{aligned}
$$

где

$$
g_{\alpha \varepsilon}(\gamma)=\int_{0}^{\infty} \alpha^{2}(C) \mathcal{E}(C) C^{4} g(C) d C
$$

Отсюда находим

$$
B^{+}=Q_{x} \frac{6 \pi^{2} \hbar^{3}}{(2 s+1) m\left(k T_{\mathrm{s}}\right)^{3} g_{\alpha \varepsilon}(\gamma)} .
$$

Таким образом, величина $\varepsilon_{0}$ имеет вид

$$
\varepsilon_{0}=Q_{x} \frac{6 \pi^{2} \hbar^{3}}{(2 s+1) m\left(k T_{\mathrm{s}}\right)^{3}} \cdot \frac{g_{\varepsilon, 2}(\gamma)}{3 g_{\varepsilon, 3}(\gamma) g_{\alpha \varepsilon}(\gamma)} .
$$

Возвращаясь к формуле для скачка температуры $\Delta T=R Q_{x}$, получаем из (25) выражение для сопротивления Капицы в нулевом приближении

$$
R=C(\gamma, q) \frac{\hbar^{3}}{(2 s+1) k^{3} T_{\mathrm{s}}^{2} m}
$$

где

$$
C(\gamma, q)=\frac{4 \pi^{2} g_{\varepsilon, 2}(\gamma)}{g_{\varepsilon, 3}(\gamma) g_{\alpha \varepsilon}(\gamma)} \cdot \frac{1+q}{1-q}
$$

- безразмерный коэффициент скачка температуры.

Положительность коэффициента при $Q_{x}$ в формуле (25) указывает на то, что стенка имеет температуру выше, чем квантовый бозе-газ.

Графики поведения коэффициента скачка температуры согласно (26) представлены на рис. 1-4. Из приведенных графиков видно, что при фиксированных значениях остальных параметров величина коэффициента $C(\gamma, q)$ :

а) монотонно убывает с ростом параметра $\gamma$;

б) монотонно растет с ростом параметра зеркальности $q$;

в) монотонно растет с ростом безразмерной величины скорости звука $w_{0}$.

Кроме того, при стремлении коэффициента зеркальности $q$ к единице величина $C(\gamma, q)$ неограниченно возрастает, так как в этом пределе теплообмен между стенкой и прилегающим к ней газом становится невозможным. 


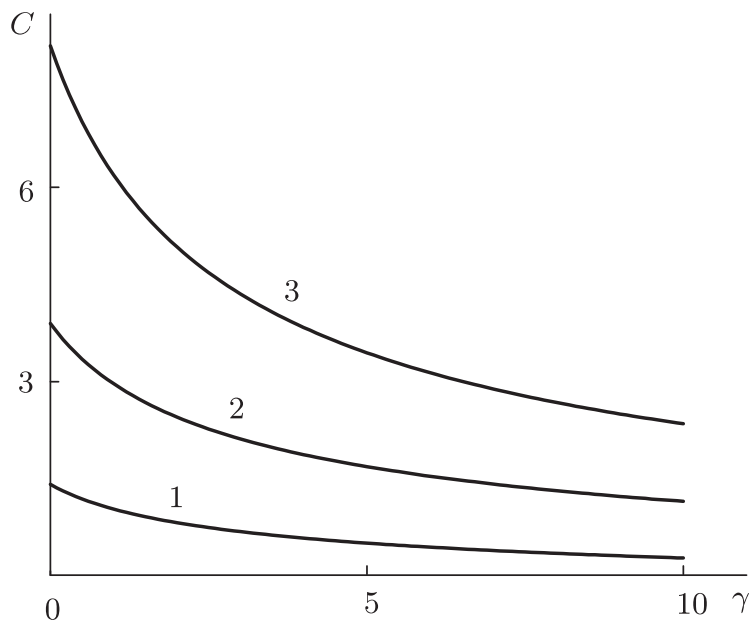

Рис. 1. Зависимость коэффициента скачка температуры от параметра $\gamma$ в случае нулевого коэффициента зеркальности $(q=0)$. Кривые $1,2,3$ отвечают значениям безразмерной скорости $w_{0}=1,2,3$.

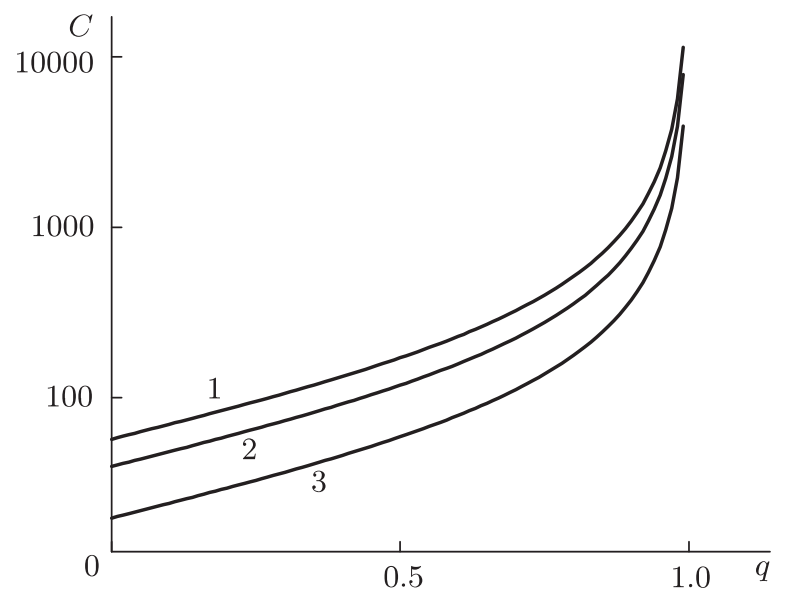

Рис. 2. Зависимость коэффициента скачка температуры от коэффициента зеркальности $q$ в случае, когда безразмерная скорость звука $w_{0}=10$. Кривые 1, 2, 3 отвечают значениям параметра столкновений $\gamma=1,3,10$. 


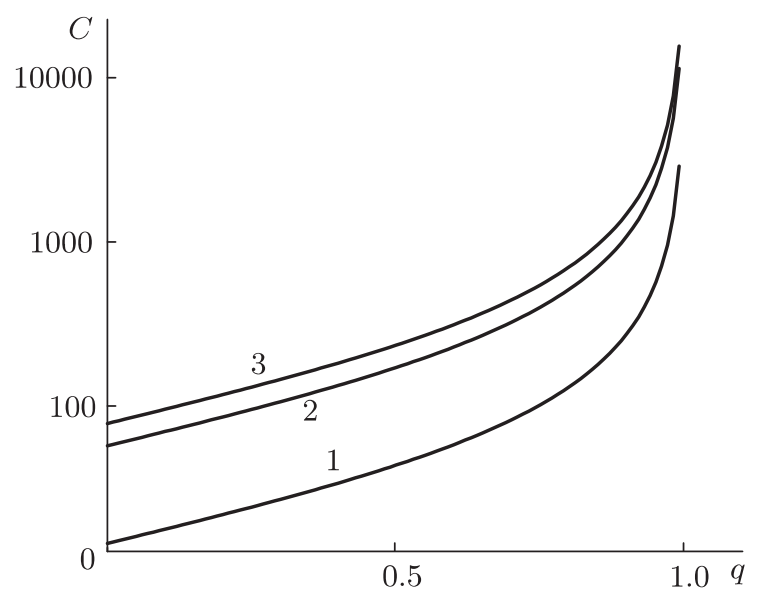

Рис. 3. Зависимость коэффициента скачка температуры от коэффициента зеркальности $q$ в случае $\gamma=1$. Кривые $1,2,3$ отвечают значениям безразмерной скорости $w_{0}=5,10,20$.

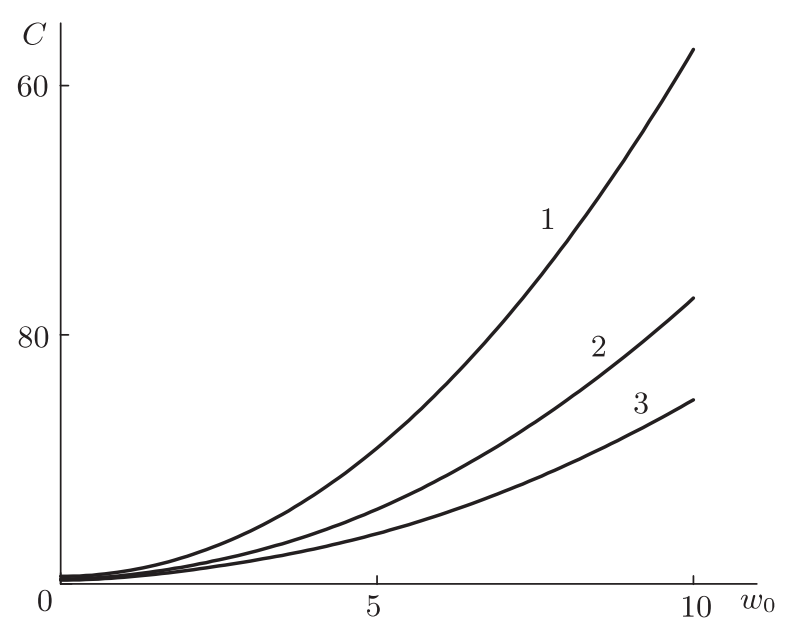

Рис. 4. Зависимость коэффициента скачка температуры от безразмерной скорости $w_{0}$ в случае $q=0.5$. Кривые $1,2,3$ отвечают значениям параметра $\gamma=1,5,10$. 


\section{8. ЗАКЛЮЧЕНИЕ}

Для вырожденного квантового бозе-газа с частотой столкновений, зависящей от импульса элементарных возбуждений бозе-газа, построено кинетическое уравнение. Рассмотрен общий случай зависимости энергии элементарных возбуждений бозе-газа от импульса. Граничные условия предполагаются зеркально-диффузными. Получено решение полупространственной граничной задачи о скачке температуры на границе вырожденного бозе-газа при наличии конденсата Бозе-Эйнштейна. Выведена формула для нахождения величины скачка температуры и вычисления сопротивления Капицы. Разработан достаточно общий метод решения кинетических уравнений с зеркально-диффузными граничными условиями, впервые предложенный в работе [17] в задаче о скин-эффекте.

\section{Список литературы}

[1] Е. М. Лифшиц, Л. П. Питаевский, Теоретическая физика. т. 10: Физическая кинетика, Наука, М., 1979.

[2] А.М. Прохоров (ред.), Физический энциклопедический словарь, Большая российская энциклопедия, М., 1995.

[3] К. Черчиньяни, Теория и приложения уравнения Больцмана, Мир, М., 1978.

[4] А. В. Латышев, ПММ, 54:4 (1990), 581-586.

[5] А. В. Латышев, А. А. Юшканов, ТМФ, 142:1 (2005), 93-111.

[6] А. В. Латышев, А. А. Юшканов, ТМФ, 161:1 (2009), 95-108.

[7] Л. П. Питаевский, УФН, 176:4 (2006), 345-364.

[8] H. Spohn, Physica D, 239:10 (2010), 627-634, arXiv: 0809.4551v1.

[9] Hai Pang, Wu-Sheng Dai, Mi Xie, J. Phys. A, 39:11 (2006), 2563-2571, arXiv: cond-mat/0603289.

[10] L. Šamaj, B. Jancovici, J. Stat. Mech., 02 (2007), P02002, 19 pp., arXiv: cond-mat/0701773.

[11] И. М. Халатников, Введение в теорию сверхтекучести, Наука, М., 1965.

[12] А. В. Латышев, А. А. Юшканов, ТМФ, 134:2 (2003), 310-324.

[13] А. В. Латышев, А. А. Юшканов, Матем. моделирование, 15:5 (2003), 80-94.

[14] Л. Д. Ландау, Е. М. Лифшиц, Теоретическая физика. т. 9: Статистическал физика. Ч. 2. Теория конденсированного состояния, Наука, М., 1978.

[15] А. В. Латышев, А. А. Юшканов, ТМФ, 155:3 (2008), 498-511.

[16] А. В. Латышев, А. А. Юшканов, ТМФ, 162:1 (2010), 112-124, arXiv: 1001.0433.

[17] А.В. Латышев, А.А. Юшканов, Ж. вычисл. матем. и матем. физ., 49:1 (2009), $137-151$. 